\title{
Case of Rapidly Expanding Conjunctival Malignant Melanoma Initially from Primary Acquired Melanosis Diagnosed 14 Years Earlier
}

Hironobu Jimura'

Yuuki Mishima'

Chie Sotozono ${ }^{2}$

Akihide Watanabe ${ }^{2}$

Jun $\mathrm{Asai}^{3}$

Chiho Ohbayashi ${ }^{4}$

Nahoko Ogata'

'Department of Ophthalmology, Nara Medical University, Nara, Japan;

${ }^{2}$ Department of Ophthalmology, Kyoto Prefectural University of Medicine, Kyoto, Japan; ${ }^{3}$ Department of Dermatology,

Kyoto Prefectural University of Medicine, Kyoto, Japan; ${ }^{4}$ Department of Diagnostic Pathology, Nara Medical University, Nara, Japan
Correspondence: Nahoko Ogata

Department of Ophthalmology, Nara

Medical University, Nara, Japan

Tel +8I-744-29-8884 (ext.3432)

Fax $+81-744-23-8032$

Email ogata@naramed-u.ac.jp

\begin{abstract}
Primary acquired melanosis (PAM) of the conjunctiva is a potentially serious melanocytic lesion that can lead to the development of a melanoma. A 60-year-old woman noticed pigmentation of the conjunctiva of her left eye for more than 10 years. She underwent excisional biopsy combined with cryotherapy and was diagnosed with PAM without atypia by intraoperative consultation. She was followed for 7 years, and no changes were observed. Fourteen years after the initial biopsy, she noted a growing conjunctival tumor, and a melanoma was suspected. She underwent orbital exenteration and skin grafting procedures. Histopathological examination of the specimen led to a diagnosis of conjunctival malignant melanoma. Re-examination of the initial biopsy specimen revealed that there was a proliferation of melanocytes that partially expanded over the basal layer of the conjunctiva which had been diagnosed as PAM with moderate atypia. We conclude that this case of conjunctival PAM had progressed to a conjunctival malignant melanoma after 14 years. Pathological evaluation of intraepithelial lesions has its limitations; thus, cases of PAM, even in the absence of obvious atypia, require careful follow-up.
\end{abstract}

Keywords: primary acquired melanosis, conjunctival malignant melanoma, malignant melanoma, conjunctival melanoma, conjunctival tumor

\section{Introduction}

Primary acquired melanosis (PAM) of the conjunctiva is a potentially serious melanocytic lesion that can lead to the development of a melanoma. ${ }^{1-4}$ A conjunctival melanoma is a rare malignant lesion of the ocular surface, and it has been estimated that approximately $75 \%$ of conjunctival melanomas arise from PAMs. $^{5-7}$ We report a case with a conjunctival PAM that developed into a conjunctival malignant melanoma after 14 years.

\section{Case Report}

A 60-years-old woman reported that she noticed pigmentation of the conjunctiva of the left eye beginning about 10 years earlier. She was suspected of having conjunctival melanoma and visited our hospital in 2006. Our initial examination found that she had no history of ocular or systemic diseases. Her decimal best-corrected visual acuity (BCVA) was 1.2, and her intraocular pressure was normal in both eyes. Slit-lamp examinations showed that the conjunctiva of the right eye was normal but diffuse disseminated pigmentation was detected around the cornea of the left eye (Figure 1). The cornea 


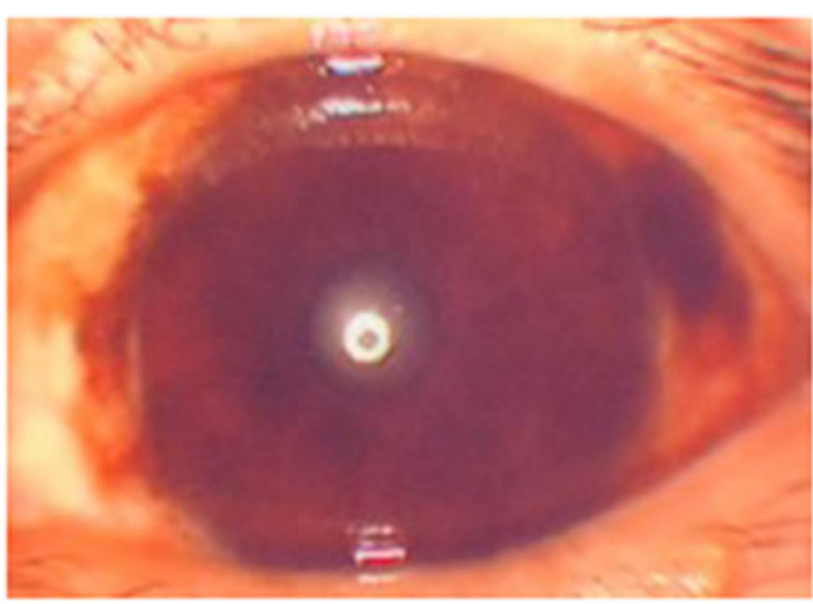

Figure I Initial slit-lamp examination of the left eye. Diffuse disseminated pigmentation in the conjunctiva can be seen around the cornea.

was clear, the anterior chamber was normal, the lens was transparent, and no abnormalities were observed in fundus of both eyes. She underwent excisional biopsy combined with cryotherapy. Histopathological analysis by frozen section suggested PAM with subepithelial reactive lymphoid hyperplasia, as there was no melanocytic nests or obvious cellular atypia and pigmentation (Figure 2). She was followed for 7 years after the biopsy and no changes were observed (Figure 3). She was then followed at the hospital near her home.

Fourteen years after the excisional biopsy, she felt discomfort of the left eye and noted a conjunctival tumor that was growing. She then consulted our hospital in 2020 . Our examination showed that her BCVA was the same; however, a black tumor that extended from the fornix and disseminated pigments at the bulbar and palpebral conjunctiva were detected in her left eye (Figure 4). A conjunctival malignant melanoma was suspected, and

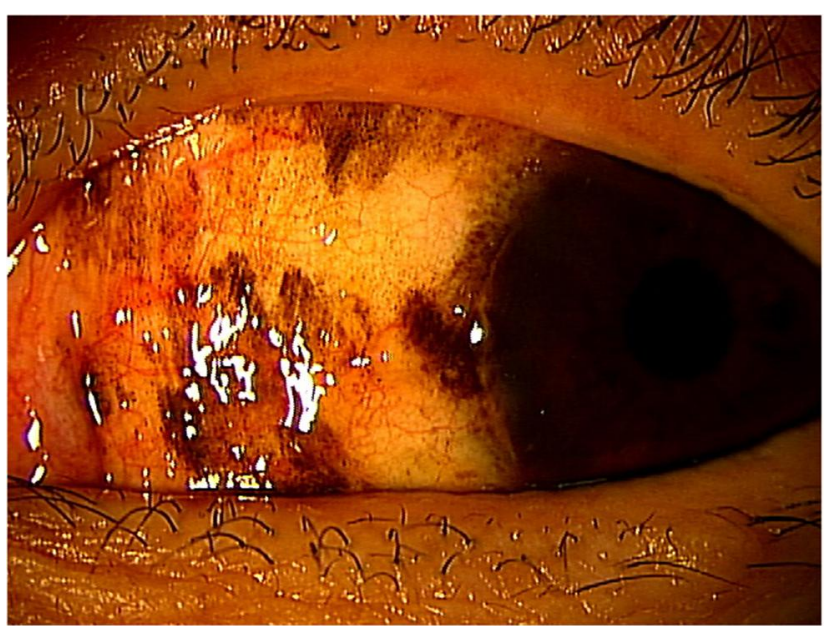

Figure 3 Seven years after excisional biopsy. No change was observed on the pigmentation in the conjunctiva.

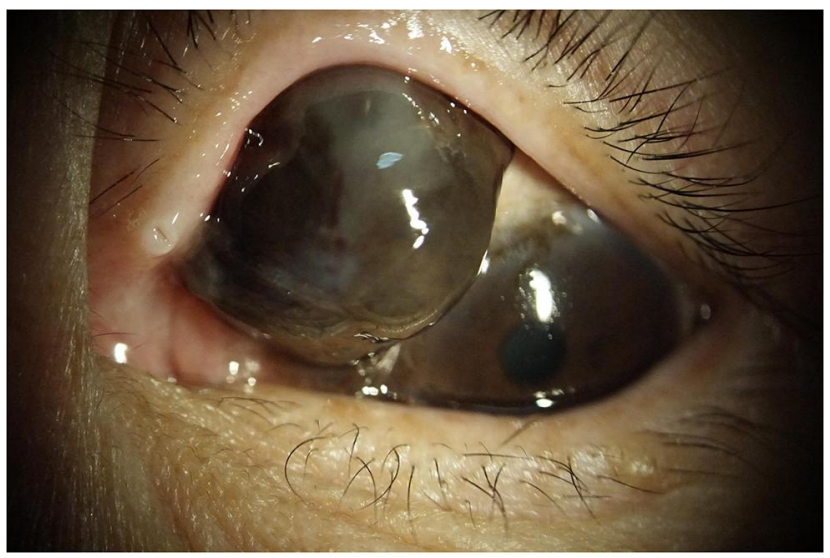

Figure 4 Fourteen years after the excisional biopsy. Black tumor expanding from the fornix and disseminated pigments at bulbar and palpebral conjunctiva can be seen.

she underwent orbital exenteration and skin grafting procedures at the Kyoto Prefectural University of Medicine Hospital. Histopathological analysis revealed that the
A

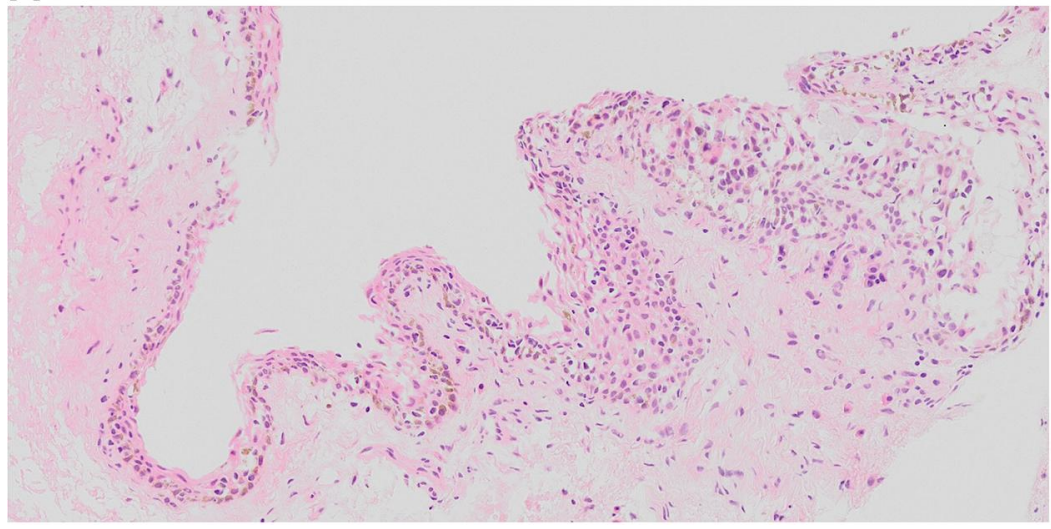

B

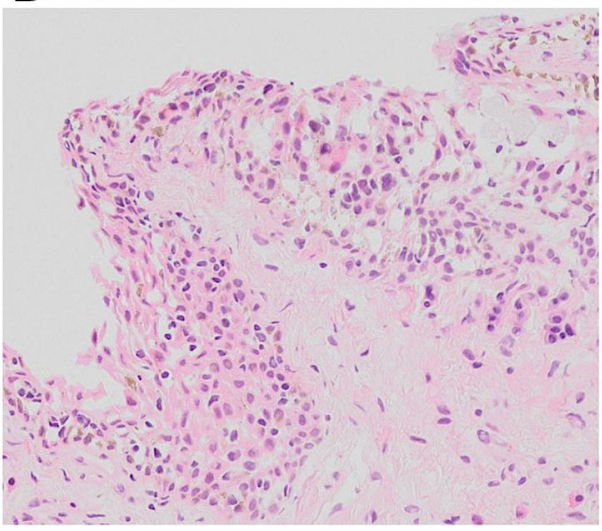

Figure 2 Rapid histopathological analysis of initial biopsy. There was no excess melanocyte proliferation in the epithelial layers. Thus, it was diagnosed as primary acquired melanosis (PAM) with reactive lymphoid hyperplasia and without atypia (A: low magnification, B; high magnification). 


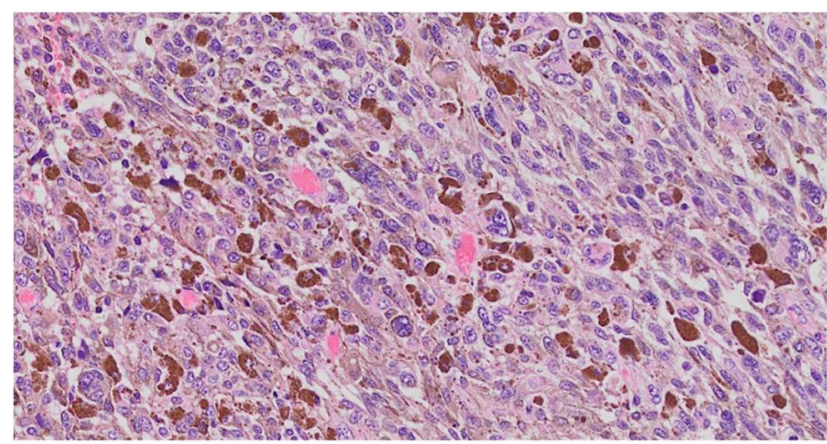

Figure 5 Histopathological specimen of the conjunctival tumor (H\&E staining) Tumor was a conjunctival malignant melanoma.

tumor was a conjunctival malignant melanoma (Figure 5) but no metastasis was found by a general check-up and her postoperative course was good.

\section{Discussion}

PAM is usually initially managed with observation alone or with excision and cryotherapy. ${ }^{8-10}$ PAM with severe atypia shows progression to melanoma in $13 \%$ to $46 \%$ of the eyes whereas PAM without atypia or with mild atypia shows $0 \%$ progression to malignant melanoma. ${ }^{1,4}$ Therefore, we prepared specimen again from a permanent formalin-fixed paraffin embedded block of the frozen section diagnosed in 2006, and confirmed inconspicuous nests of melanocytes with mild to moderate atypia extending throughout epithelial thickness. It was then diagnosed by histological scoring to be a conjunctival melanocytic intraepithelial neoplasia (C-MIN) of 2-3 (Figure 6). Thus, the diagnosis was PAM with moderate atypia.

The extent of the conjunctiva involved by PAM is the most important clinical risk factor for the progression to conjunctival melanoma. ${ }^{4,10}$ Our case was diagnosed as conjunctival PAM without atypia but the diagnosis was made from a small piece of conjunctival lesions and the other lesions might have demonstrated worse pathological findings. Although we followed up multiple pigmentary lesions carefully, these lesions were left 7 years after the initial biopsy and after then, they had developed. Thus, it might have been better to remove the remaining pigmentary lesions or to treat with topical anti-tumor eyed drops such as mitomycin c. ${ }^{10}$

In conclusion, we recommend that cautious observations and follow-up should be required for PAM.

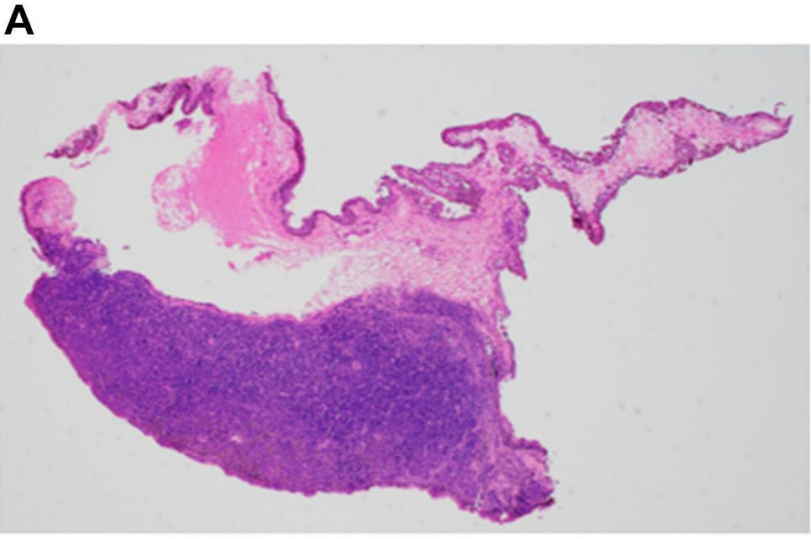

B

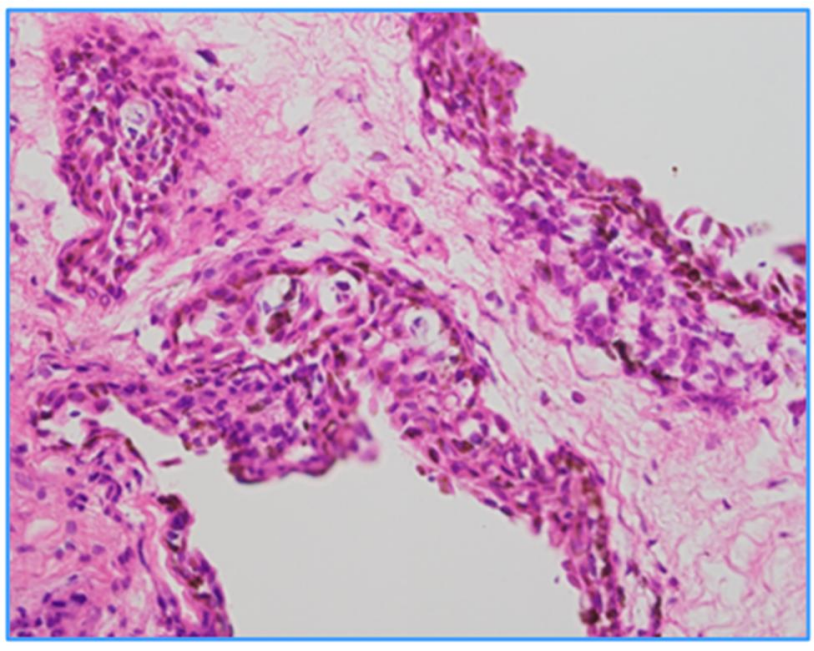

Figure 6 Re-examined of the initial excised sample. (A) There is reactive lymphoid hyperplasia (original magnification, X 20). (B) Melanocytic nests partially expanded throughout epithelial layer of the conjunctiva scoring for conjunctival melanocytic intraepithelial neoplasia (C-MIN) of 2-3 (original magnification, $X 200$ ). The diagnosis was PAM with moderate atypia.

\section{Conclusion}

Pathological evaluation of intraepithelial lesions has its limitations especially small biopsy specimen; thus, cases of PAM, even in the absence of obvious atypia, require careful follow-up.

\section{Abbreviations}

PAM, primary acquired melanosis; BCVA, best-corrected visual acuity; C-MIN, conjunctival melanocytic intraepithelial neoplasia.

\section{Data Sharing Statement}

All data supporting the conclusions of this article are included in this published article. 


\section{Consent for Publication}

Written informed consent was obtained from the patient for publication of this case report and any accompanying images. A copy of the written consent is available for review by the Editor of this journal.

\section{Acknowledgment}

Professor Duco Hamasaki of the Bascom Palmer Eye Institute for discussions and manuscript revision.

\section{Author Contributions}

All authors made a significant contribution to the work reported, whether that is in the conception, study design, execution, acquisition of data, analysis and interpretation, or in all these areas; took part in drafting, revising or critically reviewing the article; gave final approval of the version to be published; have agreed on the journal to which the article has been submitted; and agree to be accountable for all aspects of the work.

\section{Funding}

No funding or grant support.

\section{Disclosure}

Dr Chie Sotozono reports grants from Japan Agency for Medical Research and Development (AMED), Ministry of Health, Labour and Welfare, Japan, Japanese Ministry of Education, Culture, Sports, Science and Technology, and a Research Grant, outside the submitted work. The authors declare that they have no other competing interests.

\section{References}

1. Folberg R, McLean IW, Zimmerman LE. Primary acquired melanosis of the conjunctiva. Hum Pathol. 1985;16:129-135.

2. Shields CL, Demirci H, Karatza E, Shields JA. Clinical survey of 1643 melanocytic and nonmelanocytic tumors of the conjunctiva. Ophthalmology. 2004;111:1747-1754. doi:10.1016/j.ophtha.2004. 02.013

3. Shields CL, Markowitz JS, Belinsky I, et al. Conjunctival melanoma: outcomes based on tumor origin in 382 consecutive cases. Ophthalmology. 2011;118:389-395. doi:10.1016/j.ophtha.2010.06. 021

4. Shields JA, Shields CL, Mashayekhi A, et al. Primary acquired melanosis of the conjunctiva: risks for progression to melanoma in 311 eyes. The 2006 Lorenz E. Zimmerman lecture. Ophthalmology. 2008;115:511.e2-519.e2.

5. Folberg R, McLean IW, Zimmerman LE. Conjunctival melanosis and melanoma. Ophthalmology. 1984;91:673-678. doi:10.1016/S01616420(84)34245-2

6. Folberg R, McLean IW, Zimmerman LE. Malignant melanoma of the conjunctiva. Hum Pathol. 1985;16:136-143. doi:10.1016/S00468177(85)80062-9

7. Jakobiec FA, Folberg R, Iwamoto T. Clinicopathologic characteristics of premalignant and malignant melanocytic lesions of the conjunctiva. Ophthalmology. 1989;96:147-166. doi:10.1016/S01616420(89)32920-4

8. Brownstein S, Jakobiec FA, Wilkinson RD, et al. Cryotherapy for precancerous melanosis (atypical melanocytic hyperplasia) of the conjunctiva. Arch Ophthalmol. 1981;99:1224-1231. doi:10.1001/ archopht.1981.03930020098009

9. Jakobiec FA, Rini FJ, Fraunfelder FT, Brownstein S. Cryotherapy for conjunctival primary acquired melanosis and malignant melanoma: experience with 62 cases. Ophthalmology. 1988;95:1058-1070. doi:10.1016/S0161-6420(88)33058-7

10. Cohen VML, O'Day RF. Management issues in conjunctival tumours: conjunctival melanoma and primary acquired melanosis. Ophthalmol Ther. 2019;8:501-510. doi:10.1007/s40123-019-00219-8

\section{Publish your work in this journal}

The International Medical Case Reports Journal is an international, peer-reviewed open-access journal publishing original case reports from all medical specialties. Previously unpublished medical posters are also accepted relating to any area of clinical or preclinical science. Submissions should not normally exceed 2,000 words or 4 published pages including figures, diagrams and references. The manuscript management system is completely online and includes a very quick and fair peer-review system, which is all easy to use. Visit http://www.dovepress.com/testimonials.php to read real quotes from published authors. 